\title{
Integrated proteomic and metabolomic analysis to study the effects of spaceflight on Candida albicans
}

\author{
Jiaping Wang, Yu Liu, Guangxian Zhao, Jianyi Gao, Junlian Liu, Xiaorui Wu, Chong Xu and Yongzhi Li (D)
}

\begin{abstract}
Background: Candida albicans is an opportunistic pathogenic yeast, which could become pathogenic in various stressful environmental factors including the spaceflight environment. In this study, we aim to explore the phenotypic changes and possible mechanisms of $C$. albicans after exposure to spaceflight conditions.

Results: The effect of $C$. albicans after carried on the "SJ-10" satellite for 12 days was evaluated by proliferation, morphology, environmental resistance and virulence experiment. The result showed that the proliferation rate, biofilm formation, antioxidant capacity, cytotoxicity and filamentous morphology of C. albicans were increased in the spaceflight group compared to the control group. Proteomics and metabolomics technologies were used to analyze the profiles of proteins and metabolites in C. albicans under spaceflight conditions. Proteomic analysis identified 548 up-regulated proteins involved in the ribosome, DNA replication, base excision repair and sulfur metabolism in the spaceflight group. Moreover, 332 down-regulated proteins related to metabolic processes were observed. The metabolomic analysis found five differentially expressed metabolites. The combined analysis of proteomic and metabolomic revealed the accumulation of cysteine and methionine in C. albicans after spaceflight.

Conclusions: Mechanisms that could explain the results in the phenotypic experiment of C. albicans were found through proteomic and metabolomic analysis. And our data provide an important basis for the assessment of the risk that $C$. albicans could cause under spaceflight environment.
\end{abstract}

Keywords: Spaceflight, Metabolomics, Proteomics, Candida albicans

\section{Background}

In recent years, with the development of space technology, microbial space safety has become a research hotspot. It has been known that microorganisms such as bacteria and fungi have widely existed in the International Space Station [1]. C. albicans is a common conditional pathogen that usually parasitizes on human skin, mouth, urinary tract, and reproductive system [2]. While the pathogenicity of $C$. albicans may change with the changes in the external environment [3]. It has been reported that microorganisms including $C$. albicans proliferate more rapidly in the International Space Station $[4,5]$, thereby multiplying the risk of onboard crosscontamination, colonization, and infection. Worse, the space environment can potentially alter microbial

\footnotetext{
* Correspondence: liyongzhi666@sina.com

China Astronaut Research and Training Center, Beijing 100094, China
}

physiology and virulence [6]. Moreover, the immunological investigations of the astronauts recorded several dysregulations, including lymphocyte proliferation, cytokine production [7] and redistribution of leukocyte subsets [8]. Together, the presence of C. albicans poses a potential threat to astronauts' health. However, little is known about the molecular mechanism changes of $C$. albicans under the space environment.

A comprehensive understanding of the molecular communication will therefore provide new insights into this molecular mechanism. With the development of omics technology, high-throughput molecular identification and quantitation became available. Proteomics [9] could explore the expression of all the proteins of $C$. albicans under different environmental conditions. Metabolomics [10] is the study of the composition and variation of metabolic groups, thereby revealing the overall metabolic response and dynamic changes under

(c) The Author(s). 2020 Open Access This article is distributed under the terms of the Creative Commons Attribution 4.0 International License (http://creativecommons.org/licenses/by/4.0/), which permits unrestricted use, distribution, and 
different conditions. Thus, the results of integrated omics analysis could be useful for understanding the molecular mechanism of $C$. albicans with the rapid proliferation and enhanced toxicity under the space environment.

In this study, we took an integrative proteomic and metabolomic approach to identify the differentially expressed molecules of C. albicans carried by the SJ-10 satellite. The result of proteomic and metabolomic could reflect the phenotypic changes of C. albicans. To our knowledge, this is the first multi-omics approach to study $C$. albicans under spaceflight environment.

\section{Results}

Effect of $C$. albicans under space environment

After exposure to the spaceflight environment, $C$. albicans was recovered in the sabouraud-dextrose broth (SDB) medium and their survival was evaluated by $\mathrm{OD}_{600}$ measurement. The same measurements were applied towards non-exposed control C. albicans, which were cultured at the ground. As shown in Fig. 1A, the growth lag period of the spaceflight group was about $8 \mathrm{~h}$ after inoculated into medium, which was shorter than that of the control group (about $10 \mathrm{~h}$ ). The mean specific growth rate of the

\section{A}

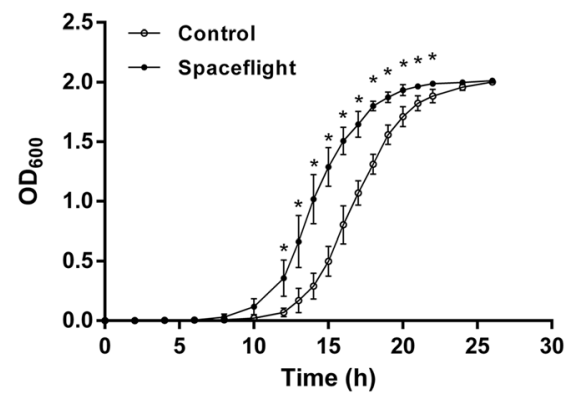

C

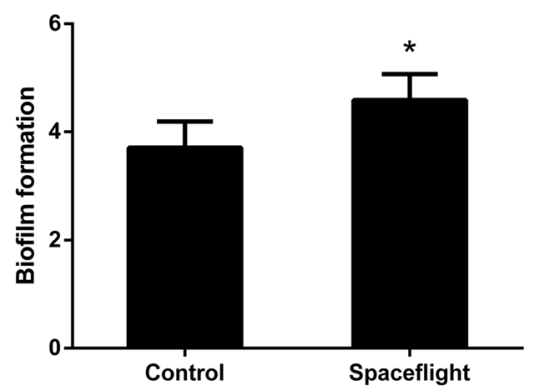

E

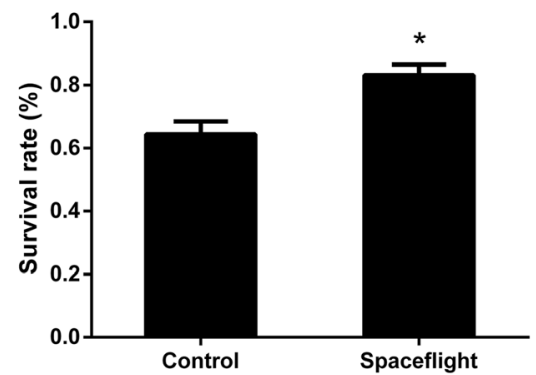

B
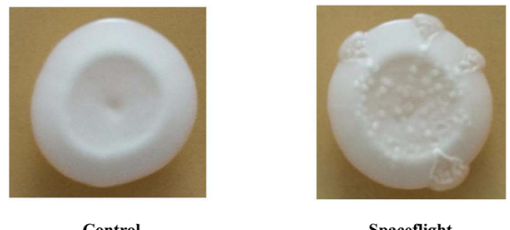

Spaceflight

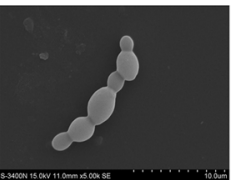

Spaceflight $(5000 \times)$

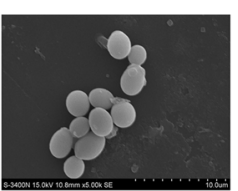

Control (5000 $\times)$
Spaceflight $(1000 \times)$

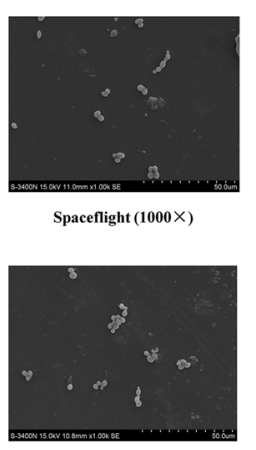

Control $(1000 \times)$

Fig. 1 Effect of $C$. albicans under spaceflight environment. a The growth curves of $C$. albicans under spaceflight environment compared with control. b Colony wrinkles of $C$. albicans after exposured to spaceflight (right) compared with control (left). c The relative biofilm formation of $C$. albicans. d Cell morphology of C. albicans under normal and spaceflight conditions. e Survival rate of C. albicans exposed to $0.0003 \%$ hydrogen peroxide under spaceflight compared with the control group. *: The $\mathrm{OD}_{600}$ of spaceflight group was significantly higher than that of control group $(P<0.05)$ at the same time after inoculation 
spaceflight group was $0.44 / \mathrm{h}$, which was faster than that of the control group $(0.35 / \mathrm{h}, P=0.018)$. As shown in Fig. 1B, the surface of C. albicans in the spaceflight group was rougher and the wrinkles at the edges were increased when compared to the control group. The amount of biofilm formation was significantly increased in the spaceflight group (Fig. 1C). Increased filamentous forms and budding with tight cell connections were observed in spaceflight $C$. albicans through scanning electron microscope (SEM), while grape-like clusters with sparse cell connections were observed in the control group (Fig. 1D). The environmental resistance evaluation result showed that the survival rate of the spaceflight group was higher than that of the control group in the SDB medium containing hydrogen peroxide (Fig. 1E). However, no significant difference was observed between two groups of $C$. albicans in resistance to acid, alkali, alcohol, and salt (Additional file 1: Figure S1). The result of virulence experiment (Additional file 2: Figure S2) showed that the spaceflight group had a lower maximum and a more significant decrease of the normalized cell index (NCI) compared to the control group, which indicates that spaceflight group has stronger cytotoxicity. All in all, the proliferation rate, biofilm formation, antioxidant capacity and cytotoxicity of $C$. albicans were increased after exposure to the spaceflight environment.

\section{Proteomic analysis of $C$. albicans}

In total, 3670 proteins were identified and 3499 proteins were quantified in the spaceflight group and control group of C. albicans by tandem mass tag (TMT) (Additional file 4: Table S1). Proteins with ratios spaceflight /control higher or lower than 1.2-fold change with adjusted $P$ value $<0.05$ were considered to be significantly changed. This resulted in 548 significantly up-regulated and 332 significantly down-regulated proteins in the spaceflight group compared with the control group (Additional file 5: Table S2), which were shown in Fig. 2A. Quantitative proteome data of C. albicans samples were used for hierarchical clustering (Fig. 2B) and principal component analysis (PCA) (Fig. 2C). The result showed that the spaceflight group and control group are clearly separated, which reflected the significant change of protein expression in C. albicans after exposure to the spaceflight environment.

To identify biological function changes in C. albicans under spaceflight environment, KEGG enrichment analysis was performed with the differentially expressed proteins (DEPs) by DAVID. The significantly enriched KEGG pathways (ranked by $P$ value) in up-regulated or down-regulated proteins were shown in Fig. 2D. Among the 548 up-regulated proteins in spaceflight group, pathways including ribosome and DNA replication were enriched, which explains the increased proliferation rate of spaceflight $C$. albicans. In addition, base excision repair was also enriched, which related to DNA damage repair and may explain the increased antioxidant capacity of the spaceflight group. Sulfur metabolism was enriched in up-regulated proteins. While metabolic processes such as carbon metabolism, biosynthesis of secondary metabolites, glyoxylate and dicarboxylate metabolism, propanoate metabolism, phenylalanine metabolism, tyrosine metabolism, and fatty acid degradation were significantly enriched in the down-regulated proteins. This reflected the complex metabolic regulation in spaceflight $C$. albicans.

\section{Metabolomic analysis of C. albicans}

To investigate the effect of spaceflight on metabolism, we used ultra-performance liquid chromatography mass spectrometry (UPLC/MS)-based metabolomics approach to untargeted quantify the metabolites in $C$. albicans. In total, 1465 peaks were identified in the spaceflight group and control group of $C$. albicans. After comparing two groups of $C$. albicans with OPLS-DA, five significantly different abundance features $(P<0.05$, VIP $>1)$ were identified (Additional file 6: Table S3). Those features were further annotated with public databases. Out of these five metabolites, $5^{\prime}$-Methylthioadenosine and adenylsuccinic acid were significantly up-regulated in the spaceflight group (Fig. 3A). While LysoPE (18:3(9Z,12Z, 15Z)/0:0), LysoPE (16:1(9Z)/0:0) and LysoPE (18:2(9Z, $12 Z) / 0: 0)$ were significantly down-regulated in spaceflight group (Fig. 3B).

\section{Integrated analysis of proteome and metabolome data in C. albicans}

To integrate the result of proteome and metabolome data, we transformed protein ID and metabolites name to KEGG ID and KEGG compound, respectively. After mapping molecular objects to KEGG pathway, three pathways including purine metabolism, cysteine and methionine metabolism, and alanine, aspartate glutamate metabolism were found to be regulated by both DEPs and differentially expressed metabolites (DEMs) (Additional file 3: Figure S3). Among those three pathways, adenylsuccinic acid, which was up-regulated in spaceflight group, was mapped both in alanine, aspartate glutamate metabolism, and purine metabolism pathway. Consistent with the metabolome result, adenylosuccinate synthetase, which catalyzes IMP and L-aspartate to generate adenylsuccinic acid, was also up-regulated $(P=0.0001$, fold change $=1.17$ ) in spaceflight group from proteome data. 5'-Methylthioadenosine (MTA) is a naturally occurring sulfur-containing nucleoside, which is a 


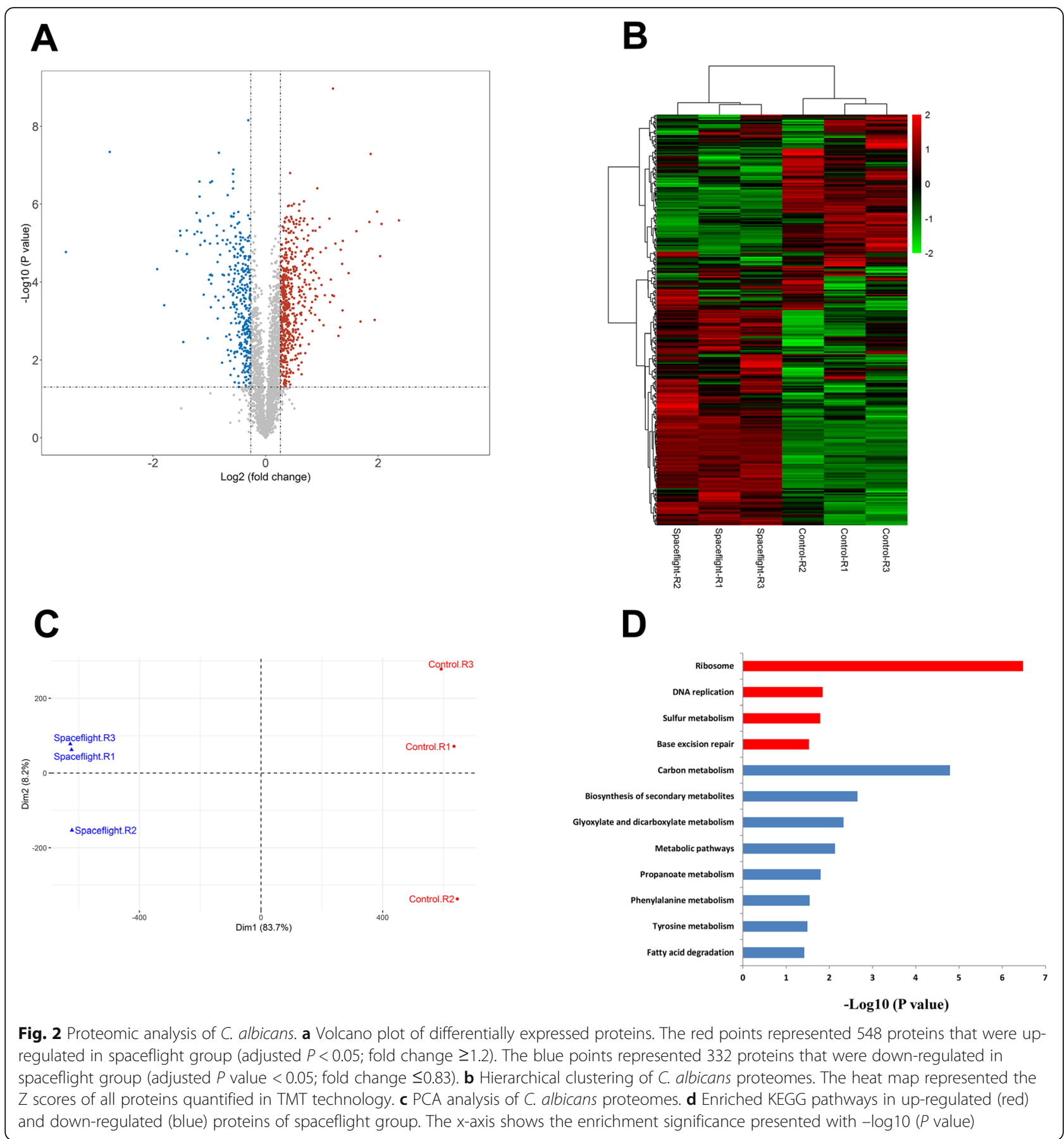

major by-product of polyamine biosynthesis involved in cysteine and methionine metabolism pathway [11]. We found MTA was up-regulated in the spaceflight group with metabolomics analysis. While S-methyl-5' thioadenosine phosphorylase (MEU1), which involved in the breakdown of MTA and responsible for the first step in the methionine salvage pathway after MTA has been generated from S-adenosylmethionine [12], was significantly up-regulated in spaceflight group of proteomics.

\section{Discussion}

C. albicans is an opportunistic pathogenic yeast [13], which usually exists as a commensal organism but can become pathogenic in immunocompromised individuals under a variety of conditions [14]. Long term spaceflight with microgravity and motionless may have adverse effects on the immune system of astronauts [7]. Meanwhile, Jiang et al. [15] reported that simulated microgravity promoted the growth rate of $C$. albicans significantly. Altenburg et al. [16] found that the growth 


\section{A}
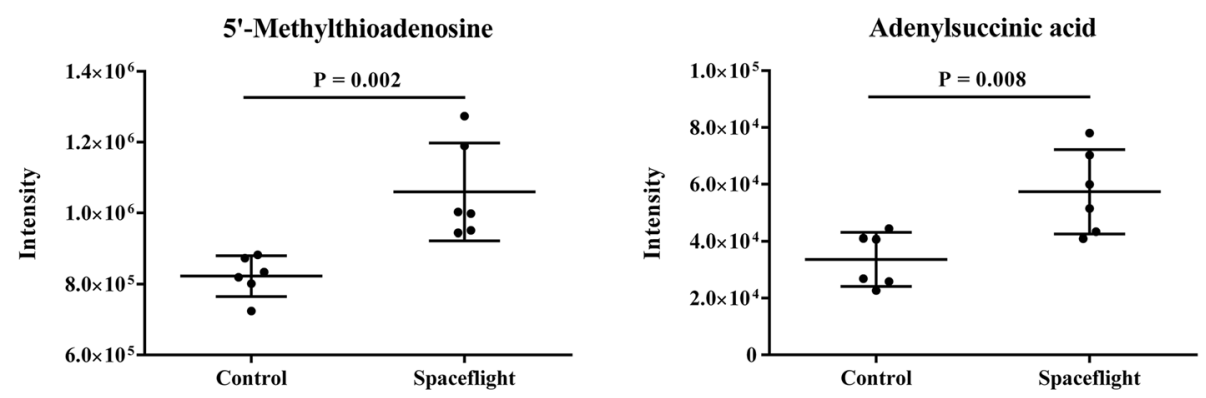

B
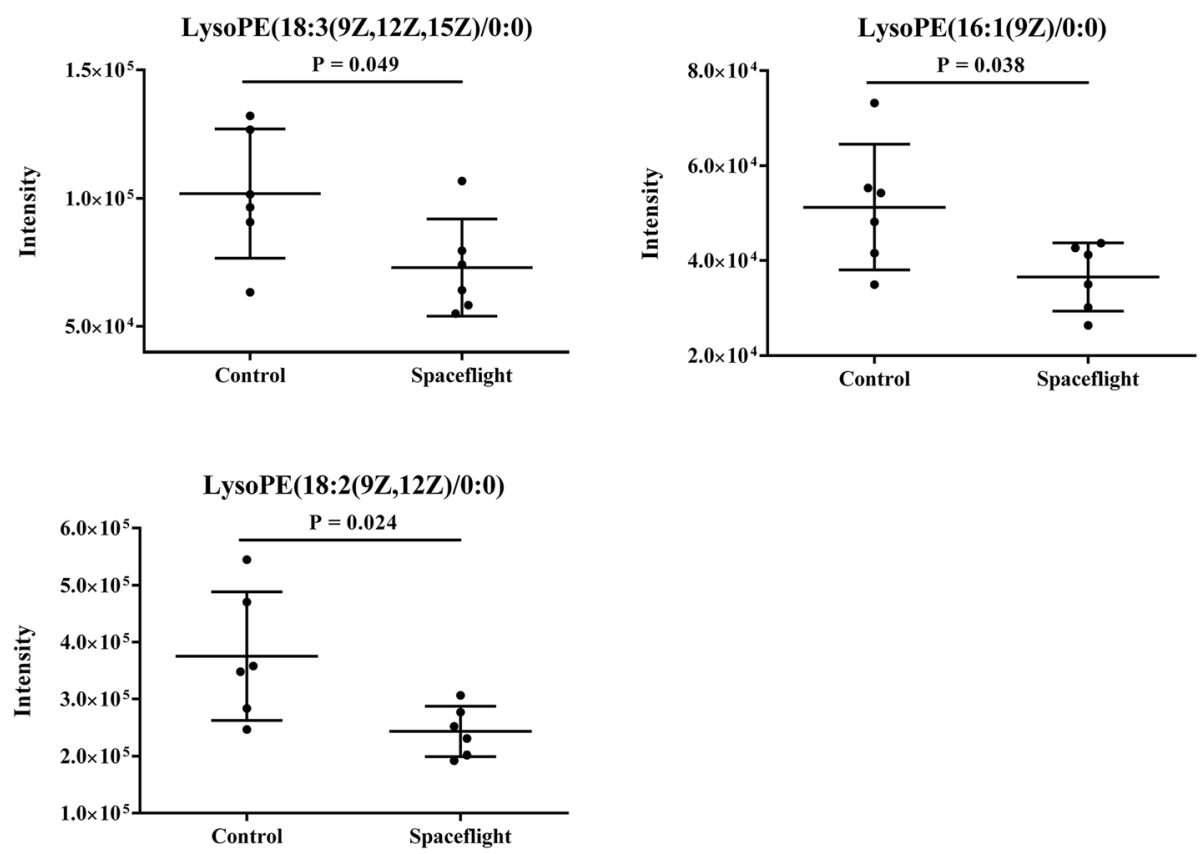

Fig. 3 Differential metabolites of C. albicans. a Scatter plots of two up-regulated metabolites in the spaceflight group. b Scatter plots of three down-regulated metabolites in the spaceflight group. $P$ value was calculated with unpaired Student's t-test

of C. albicans in simulated microgravity results in an increase in filamentous forms of the organism, which is consistent with enhanced pathogenicity. Those reports indicated that the existence of $C$. albicans in the space environment might be threated to the health of astronauts. And the specific mechanisms of C. albicans' increased proliferation and pathogenicity need further study. In this study, the combination of proteomic and metabolomic approach was used to study the effects of spaceflight on $C$. albicans. To our knowledge, this is the first report of $C$. albicans under spaceflight conditions with proteomic and metabolomic analyses.

We explored the phenotypic change of C. albicans after exposure to the spaceflight environment. Increased proliferation rate and filamentous morphology of $C$. albicans in spaceflight groups were observed, which were consistent with the previous report $[15,16]$. In addition, we found that the biofilm relative formation, antioxidant capacity, and cytotoxicity of $C$. albicans were increased in the spaceflight group. Crabbé et al. [17] reported that genes involved in oxidative stress resistance were up-regulated in spaceflight cultured $C$. albicans with microarray analysis, which was consistent with our result. However, Hammond et al. [18] reported that the virulence of $C$. albicans to kill wild type Caenorhabditis elegans was reduced under spaceflight environment. The difference between their results and ours may because their experiment was done in the International Space Station with $48 \mathrm{~h}$, while our spaceflight environment was low earth orbit flight for 12 days. The difference in space flight time and environment may have different influences on the microorganism. 
Metabolomics only identified five DEMs. There may be two reasons for this result. First, we only used the LC-based approach to detect metabolites, which leads to the missing identification of less polar metabolites. Second, high abundance metabolites such as lipid metabolites were identified, while low-abundance DEMs may be lost. Although our results may lose many metabolites, the five DEMs could be used for further combined analysis. Integrated analysis of proteomic and metabolomic of $C$. albicans revealed that spaceflight may have an influence on cysteine and methionine metabolism pathway. On the one hand, we found that S-adenosylmethionine synthase (SAM2), which catalyzes the formation of S-adenosylmethionine from methionine, was increased in spaceflight group. This was consistent with the up-regulation of MTA, which is synthesized from Sadenosylmethionine. However, the expression of MEU1, which involved in the methionine salvage pathway by breakdown MTA, was increased in the spaceflight group. This may reduce the consumption of methionine. Meanwhile, 5-methyltetrahydropteroyltriglutamate homocysteine methyltransferase (MET6), which involved in the formation of methionine, was also up-regulated (adjust $P<0.001$, fold change $=1.13$ ). On the other hand, bifunctional cysteine synthase (MET15) [19], which has cysteine synthase activity and may synthase cysteine from serine, was increased in spaceflight group. Meanwhile, aspartate aminotransferase (AAT21) and aspartate transaminase (AAT1), which may play roles in catalyzes cysteine [20], were down-regulated in spaceflight group. So we concluded that cysteine and methionine were accumulated in the spaceflight group of $C$. albicans. Besides, methionine and cysteine are sulfurcontaining amino acids, and proteins involved in sulfur metabolism such as sulfite reductase subunit alpha (MET10), sulfite reductase beta subunit, sulfate adenylyltransferase (MET3) and adenylyl-sulfate kinase (MET14) were simultaneously up-regulated in spaceflight group. Interestingly, many research reported $[21,22]$ that most genes in methionine and cysteine biosynthesis pathway were up-regulated during biofilm formation. In addition, Li et al. [23] showed that the development of $C$. albicans was enhanced by addition of methionine and cysteine. The result inferred from the proteomic and metabolomic analysis was consistent with the observed influence on phenotypic of C. albicans after spaceflight.

\section{Conclusions}

We explored the effect of spaceflight on $C$. albicans by combining proteomic and metabolomic analyses in this study. To our knowledge, this is the first proteomic and metabolomic study on C. albicans after spaceflight. Increased proliferation rate, biofilm formation, antioxidant capacity, cytotoxicity, and filamentous morphology were observed in the spaceflight group of $C$. albicans compared to the control group. Proteomic analysis identified
3670 proteins and showed that spaceflight samples and control samples could be separated from proteomic data. Enrichment analysis with DEPs indicated that proteins in the ribosome, DNA replication, base excision repair, and sulfur metabolism were significantly up-regulated, while proteins in many metabolic processes were significantly down-regulated. Metabolomic analyses found five DEMs. The combined analysis of proteomic and metabolomic revealed the accumulation of cysteine and methionine in C. albicans after spaceflight. This study showed that proteomic and metabolomic are useful tools that could explain the phenotypic changes of $C$. albicans. And the data in this study will facilitate the future mechanism exploration and disease prevention of C. albicans.

\section{Materials and method}

\section{Strains and growth conditions}

C. albicans (CMCC(F)98,001) were purchased from China General Microbiological Culture Collection Center. SDB was purchased from Oxoid. C. albicans was cultured in exponential phase $\left(\mathrm{OD}_{600}=1\right)$ and inoculated into SDB. After incubated at $30^{\circ} \mathrm{C}$ for $30 \mathrm{~h}$, half of the samples were cultured in space for 12 days carried by the "SJ-10" satellite. And the rest samples were cultured at the ground as control. Strains were preserved by adding glycerin and stored at $-20{ }^{\circ} \mathrm{C}$. Due to the limitations of the cycle and conditions of spaceflight experiments, all replicates were obtained by the recovery of experimental strain.

\section{Growth curves of $C$. albicans}

C. albicans in the spaceflight group and control group were recovered on the SDB medium at $30^{\circ} \mathrm{C}$ overnight, respectively. Samples were inoculated into SDB liquid medium and incubated at $150 \mathrm{rpm}$ at $30{ }^{\circ} \mathrm{C}$. The growth curves of $C$. albicans were recorded by using a spectrophotometer. The initial $\mathrm{OD}_{600}$ measurement is measured every $2 \mathrm{~h}$ at the beginning of the lag phase and is measured every $1 \mathrm{~h}$ in the exponential phase. Specific growth rate was calculated as the max value of $\Delta \log 10\left(\mathrm{OD}_{600}\right) /$ $\Delta$ time in the exponential phase.

\section{Colony wrinkles and biofilm formation assay}

Preserved strains of $C$. albicans $(5 \mu \mathrm{L})$ were inoculated into SDB solid medium and incubated at $30{ }^{\circ} \mathrm{C}$ for five h. Colony wrinkles of $C$. albicans were observed with a microscope.

Preserved strains of $C$. albicans $(20 \mu \mathrm{L})$ were added into $2 \mathrm{~mL}$ SDL liquid medium in glass tubes and incubated at $150 \mathrm{rpm}$ at $30{ }^{\circ} \mathrm{C}$ until the stationary phase. $\mathrm{OD}_{600}$ was measurement after incubation and the glass tubes were washed two times with deionized water gently. Glass tubes were added with $5 \mathrm{~mL} 0.1 \%$ crystal violet solution and dyeing for $15 \mathrm{~min}$ after drying $1 \mathrm{~h}$ at $60^{\circ} \mathrm{C}$. 
After washed with deionized water gently, $5 \mathrm{~mL}$ dimethyl sulfoxide was added into the glass tubes and $\mathrm{OD}_{570}$ was measured $1 \mathrm{~h}$ later. Biofilm formation was calculated as $100 \times \mathrm{OD}_{570} / \mathrm{OD}_{600}$.

\section{Morphology of C. albicans}

Preserved strains of C. albicans $(1 \mu \mathrm{L})$ were recovered with $5 \mathrm{~mL}$ SDB liquid medium at $150 \mathrm{rpm}$ at $30^{\circ} \mathrm{C}$ overnight. Then coverslips were placed in a solution mixed with sample and SDB liquid medium (1:2). After incubation $8 \mathrm{~h}$ at $30^{\circ} \mathrm{C}$, coverslips were fixed with $5 \%$ glutaraldehyde solution at $4{ }^{\circ} \mathrm{C}$ overnight. Then samples were sent to the China Academy of Chinese Medical Sciences for SEM analysis. Briefly, samples were fixed with tannic acid for $1 \mathrm{~h}$. Then samples were dehydrated by a graded series of ethanol (50, 70, 90, and 100\%) for $15 \mathrm{~min}$ at each step, and transferred to tert-butanol for $30 \mathrm{~min}$. In the end, the samples were dehydrated with dryer and coated with gold-palladium. The morphology of C. albicans was observed in Hitachi S-3400 N SEM.

\section{Environmental resistance assay}

Preserved strains of $C$. albicans $(1 \mu \mathrm{L})$ were inoculated into SDB solid medium and incubated at $30^{\circ} \mathrm{C}$ overnight. Recovered samples were inoculated into SDB liquid medium and incubated at $150 \mathrm{rpm}$ at $30^{\circ} \mathrm{C}$ until the exponential phase $\left(\mathrm{OD}_{600}=1\right)$. Then samples were added into five SDB liquid mediums (1:100) containing HCL (pH 3.5), ammonia ( $\mathrm{pH}$ 9.5), 3\% ethanol, $0.0003 \%$ hydrogen peroxide or $450 \mathrm{mmol} / \mathrm{L} \mathrm{NaCl}$, respectively. While normal SDB liquid medium was used as a background. After incubated $7 \mathrm{~h}$ at $200 \mathrm{rpm}$ at $30^{\circ} \mathrm{C}$, samples were diluted with PBS at three volume ratios $\left(10^{-3}, 10^{-4}\right.$ and $10^{-5}$ ). And $200 \mu \mathrm{L}$ diluted samples were evenly spread to SDB solid medium, respectively. The number of colonies was calculated after incubation at $30^{\circ} \mathrm{C}$. The survival rate was calculated as the mean number of colonies from mixed SDB divide by the mean number of colonies from normal SDB.

\section{Virulence of $C$. albicans}

LoVo cells were maintained in DMEM-F12 growth medium containing $10 \% \mathrm{FBS}$ at $37^{\circ} \mathrm{C}$ in a $\mathrm{CO}_{2}$ incubator. Cells were harvest with trypsin and adjusting concentration to $2 \times 10^{5}$ cells $/ \mathrm{mL}$. C. albicans was recovered with SDB liquid medium at $150 \mathrm{rpm}$ at $30^{\circ} \mathrm{C}$ overnight. After centrifugation at $4000 \mathrm{rpm}$ for $5 \mathrm{~min}$, the precipitation was resuspended with PBS. After centrifugation again, the precipitation was resuspended with DMEMF12 medium at the concentration of $4.5 \times 107 \mathrm{CFU} / \mathrm{mL}$. Then $100 \mu \mathrm{L}$ C. albicans were added into $200 \mu \mathrm{L}$ LoVo cells. Samples were detected with real time cell analyzer (RTCA) in a $\mathrm{CO}_{2}$ incubator for $10 \mathrm{~h}$ at $37^{\circ} \mathrm{C}$.

\section{Sample preparation for proteome and metabolome analysis}

C. albicans in spaceflight group and control group were recovered on SDB solid medium at $30{ }^{\circ} \mathrm{C}$ overnight, respectively. Recovered samples were inoculated into SDB liquid medium and incubated at $150 \mathrm{rpm}$ at $30{ }^{\circ} \mathrm{C}$ and grown to an $\mathrm{OD}_{600}$ of 1 . Samples were harvested by centrifugation at $4000 \mathrm{rpm}$ for $5 \mathrm{~min}$ at $4{ }^{\circ} \mathrm{C}$. The precipitate was collected and washed with PBS three times. All samples were stored at $-80^{\circ} \mathrm{C}$ until use.

\section{Proteome analysis}

Samples were resuspended with lysate buffer (7 M urea, $2 \mathrm{M}$ thiourea, $40 \mathrm{mM}$ DTT, $1 \mathrm{mM}$ PMSF [24]) and were sonicated ( $1 \mathrm{~s} / 1 \mathrm{~s}$ intervals, $80 \mathrm{~W}$ power) for $3 \mathrm{~min}$. Cell debris was removed by centrifugation at $3000 \mathrm{~g}$ for 5 $\min$ at $4{ }^{\circ} \mathrm{C}$. Protein concentration was determined by Bradford assay and aliquoted to store at $-80^{\circ} \mathrm{C}$. For each group, $100 \mu \mathrm{g}$ of proteins were mixed with $120 \mu \mathrm{L}$ reducing buffer $(10 \mathrm{mM}$ DTT, $8 \mathrm{M}$ Urea, $100 \mathrm{mM}$ triethylammonium bicarbonate (TEAB), $\mathrm{pH}$ 8.0) in Ami$\operatorname{con}^{\ominus}$ Ultra-0.5 Centrifugal Filter $(10 \mathrm{kDa})$ [25] and incubated at $60^{\circ} \mathrm{C}$ for $1 \mathrm{~h}$. Then iodoacetamide was added to the solution with the final concentration of $50 \mathrm{mM}$ and incubated for $40 \mathrm{~min}$ at room temperature in the dark. After centrifugation at $12000 \mathrm{rpm}$ for $20 \mathrm{~min}$, samples were washed three times with TEAB and digested with trypsin (Promega, Madison, WI, USA) (enzyme to protein ratio $1: 50$ ) at $37^{\circ} \mathrm{C}$ overnight. Digested peptides of three technical replicates per group were labeled with TMT reagents (Thermo Fisher Scientific) according to the manufacturer's instructions. For 6-plex TMT, spaceflight group samples were labeled with TMT tags 126, 127, 128, and control samples were labeled with TMT tags 129, 130, and 131, respectively. Equal amounts of TMT-labeled peptides were mixed and dried, then resuspended in buffer A (2\% acetonitrile, $98 \%$ water with ammonia at $\mathrm{pH} 10$ ) and fractionated to 15 fractions with 1100 HPLC System (Agilent Technologies, USA).

Peptides were redissolved with $0.1 \%$ formic acid (FA) and analyzed on a Q-Exactive HF mass spectrometer (Thermo Fisher Scientific, USA) coupled with a nanospray Flex source (Thermo Fisher Scientific, USA). Samples were loaded and separated by a C18 column (15 $\mathrm{cm} \times 75 \mu \mathrm{m}$ ) on an EASY $-\mathrm{nLC}^{\mathrm{rm}} 1200$ system (Thermo Fisher Scientific, USA). The flow rate was $300 \mathrm{~nL} / \mathrm{min}$ and linear gradient was $90 \mathrm{~min}$. The mass spectrometer was operated in the data-dependent mode with positive polarity at electrospray voltage of $2 \mathrm{kV}$. Full scan MS spectra (m/z 300-1600) were acquired in the orbitrap with the resolution as $70 \mathrm{~K}$, the automatic gain control (AGC) target was $1 \mathrm{e} 6$ and the maximum injection time was $80 \mathrm{~ms}$. The top 10 intense ions were isolated for HCD MS/MS fragmentation. In MS2, the resolution was 
17,500 and the AGC target was 2e5. Fragmentation was performed with normalized collision energy (NCE) of $32 \%$ and dynamic exclusion duration of $15 \mathrm{~s}$.

The mass spectrometry (MS) raw data were analyzed with Proteome Discoverer software (version 2.2) using the Sequest search engine to search against the UniProt C. albicans database. The following parameters were applied: precursor mass tolerance was $10 \mathrm{ppm}$; fragment tolerance was $0.02 \mathrm{Da}$; the dynamic modifications were oxidation (M); the static modification was carbamidomethyl $(\mathrm{C})$ and TMT labeling of amines and lysine; a maximum of two missed cleavages was allowed. Peptides with FDR $<0.01$ (based on the target-decoy database algorithm [26]) were used for protein grouping.

\section{Metabolome analysis}

Metabolites were extracted with $300 \mu \mathrm{L}$ methanol and ultrasonic oscillation for $10 \mathrm{~min}$. The supernatant was collected after centrifugation at $12000 \mathrm{rpm}$ for $10 \mathrm{~min}$ at $4{ }^{\circ} \mathrm{C}$. UPLC-QTOF/MS (Agilent Technologies, USA) was implemented for metabolites detection with six technical replicates. The experimental quality was evaluated by quality control (QC) samples. Features alignment, picking, and identification were performed by Progenesis QI (Waters, Nonlinear Dynamics, Newcastle, UK). By combining the univariate and multivariate statistical analysis, significantly changed features $(P$ value $<0.05$, VIP $>1$ ) were acquired. Those features were further annotated by Progenesis QI with public databases including Metlin, LIPID MAPS, PubChem, YMDB (Yeast Metabolome Database) and KEGG.

\section{Statistical analysis}

Perseus software [27] was used to calculate the DEPs with unpaired Student's t-test. Multiple hypothesis testing is performed using Benjamini-Hochberg correction at 5\% significance level for both DEPs calculations and subsequent KEGG enrichment analysis. SIMCA (Version 14.1) was used for statistical analysis of metabolomics data with OPLS-DA (orthogonal projections to latent structures discriminant analysis). For other results, SPSS software package (Version 16.0) was used for statistical analysis with unpaired Student's t-test. The data are presented as means \pm SD. $P<0.05$ was defined as statistical significance.

\section{Supplementary information}

Supplementary information accompanies this paper at https://doi.org/10. 1186/s12864-020-6476-5.

Additional file 1: Figure S1. Survival rate of Candida albicans under spaceflight environment compared with control. (A) Candida albicans cultured in SDB liquid mediums with $\mathrm{HCL}$ ( $\mathrm{pH}$ 3.5), (B) ammonia ( $\mathrm{pH}$ 9.5), (C) $3 \%$ ethanol, or (D) $450 \mathrm{mmol} / \mathrm{L} \mathrm{NaCl}$.
Additional file 2: Figure S2. Virulence of Candida albicans toward LoVo cells in spaceflight and ground control. Recovered Candida albicans was added into LoVo cells and the samples were detected with real time cell analyzer. Normalized cell index (NCl) was used to estimate the percentage change in adhesion, which reflected the virulence of Candida albicans

Additional file 3: Figure S3. Integrated analysis of proteome and metabolome result on KEGG pathway. (A) Purine metabolism. (B) Alanine, aspartate glutamate metabolism. (C) Cysteine and methionine metabolism. Boxes represented for proteins and dots represented for chemical compounds. The red color represented proteins or metabolites that were up-regulated in spaceflight group. The blue color represented proteins or metabolites that were down-regulated in spaceflight group.

Additional file 4: Table S1. List of proteins identified and quantified with TMT.

Additional file 5: Table S2. List of differentially expressed proteins between the spaceflight and control group.

Additional file 6: Table S3. List of differentially expressed metabolites between the spaceflight and control group.

\section{Abbreviations}

AAT1: Aspartate transaminase; AAT21: Aspartate aminotransferase; AGC: Automatic gain control; MET10: Sulfite reductase subunit alpha; MET14: Adenylyl-sulfate kinase; MET15: Bifunctional cysteine synthase; MET3: Sulfate adenylyltransferase; MEU1: S-methyl-5'-thioadenosine phosphorylase; MS: Mass spectrometry; MTA: 5'-Methylthioadenosine; NCE: Normalized collision energy; NCl: Normalized cell index; OPLSDA: Orthogonal projections to latent structures discriminant analysis; PCA: Principal Component Analysis; RTCA: Real time cell analyzer; SDB: Sabouraud dextrose broth; SEM: Scanning electron microscope; TMT: Tandem mass tag; UPLC/MS: Ultra-performance liquid chromatography mass spectrometry; YMDB: Yeast Metabolome Database

\section{Acknowledgements}

We thank Shanghai LuMing Biological Technology Co. Ltd. for proteome detection and data analysis.

\section{Authors' contributions}

JW performed the bioinformatics analysis and wrote the paper. $Y L$ and GZ performed the experiments and analyzed the data. JG, JL, XW and CX conceived the experiments and participated in discussion data. TL designed the experiments and revised the manuscript. All authors have read and approved the manuscript.

\section{Funding}

This work was supported by National Significant Science Foundation (2015ZX09J15102), 1226 Engineering Health Major Project (AWS16J018) and 1226 Engineering Health Key Project (BWS17J028). The funding bodies played no role in the design of the study and collection, analysis, and interpretation of data and in writing the manuscript.

\section{Availability of data and materials}

The mass spectrometry proteomics data have been deposited to the ProteomeXchange Consortium (http://proteomecentral.proteomexchange.org) via the iProX partner repository [28] with the dataset identifier PXD016983.

Ethics approval and consent to participate Not applicable.

Consent for publication

Not applicable.

Competing interests

The authors declare that they have no competing interests. 
Received: 11 November 2019 Accepted: 9 January 2020

Published online: 17 January 2020

\section{References}

1. Novikova N, De Boever P, Poddubko S, et al. Survey of environmental biocontamination on board the international Space Station [J]. Res Microbiol. 2006;157(1):5-12

2. Mccullough MJ, Ross BC, Reade PC. C. Albicans: a review of its history, taxonomy, epidemiology, virulence attributes, and methods of strain differentiation [J]. Int J Oral Maxillofac Surg. 1996;25(2):136-44.

3. Mayer FL, Wilson D, Hube B. C. albicans pathogenicity mechanisms [J]. Virulence. 2013;4(2):119-28.

4. Rosenzweig JA, Abogunde O, Thomas K, et al. Spaceflight and modeled microgravity effects on microbial growth and virulence [J]. Appl Microbiol Biotechnol. 2010;85(4):885-91.

5. Sugita T, Yamazaki T, Makimura K, et al. Comprehensive analysis of the skin fungal microbiota of astronauts during a half-year stay at the international Space Station [J]. Med Mycol. 2016;54(3):232-9.

6. Klaus DM, Howard HN. Antibiotic efficacy and microbial virulence during space flight [J]. Trends Biotechnol. 2006;24(3):131-6.

7. Sonnenfeld G, Butel JS, Shearer WT. Effects of the space flight environment on the immune system [J]. Rev Environ Health. 2003;18(1):1-17.

8. Crucian B, Stowe RP, Mehta S, et al. Alterations in adaptive immunity persist during long-duration spaceflight [J]. NPJ Microgravity. 2015;1:15013.

9. Prasath KG, Sethupathy S, Pandian SK. Proteomic analysis uncovers the modulation of ergosterol, sphingolipid and oxidative stress pathway by myristic acid impeding biofilm and virulence in C. albicans [J]. J Proteome. 2019:208:103503

10. Li L, Liao Z, Yang Y, et al. Metabolomic profiling for the identification of potential biomarkers involved in a laboratory azole resistance in C. albicans [J]. PLoS One. 2018;13(2):e0192328.

11. Avila MA, Garcia-Trevijano ER, Lu SC, et al. Methylthioadenosine [J]. Int J Biochem Cell Biol. 2004;36(11):2125-30.

12. Pirkov I, Norbeck J, Gustafsson L, et al. A complete inventory of all enzymes in the eukaryotic methionine salvage pathway [J]. FEBS J. 2008;275(16): 4111-20.

13. Gow NAR, Yadav B. microbe Profile: C. albicans: a shape-changing, opportunistic pathogenic fungus of humans [J]. Microbiology. 2017;163(8): 1145-7.

14. Erdogan A, Rao SS. Small intestinal fungal overgrowth [J]. Curr Gastroenterol Rep. 2015;17(4):16.

15. Jiang $W, X u B$, Yi Y, et al. Effects of simulated microgravity by RCCS on the biological features of C. albicans [J]. Int J Clin Exp Pathol. 2014;7(7):3781-90.

16. Altenburg SD, Nielsen-Preiss SM, Hyman LE. Increased filamentous growth of C. albicans in simulated microgravity [J]. Genomics Proteomics Bioinformatics. 2008:6(1):42-50.

17. Crabbe A, Nielsen-Preiss SM, Woolley CM, et al. Spaceflight enhances cell aggregation and random budding in C. albicans [J]. PLoS One. 2013;8(12): e80677

18. Hammond TG, Stodieck L, Birdsall HH, et al. Effects of microgravity on the virulence of listeria monocytogenes, enterococcus faecalis, C. albicans, and methicillin-resistant Staphylococcus aureus [J]. Astrobiology. 2013;13(11): 1081-90.

19. Viaene J, Tiels $P$, Logghe $M$, et al. MET15 as a visual selection marker for $C$ albicans [J]. Yeast. 2000;16(13):1205-15.

20. Cooper AJ, Bruschi SA, Iriarte A, et al. Mitochondrial aspartate aminotransferase catalyses cysteine S-conjugate beta-lyase reactions [J]. Biochem J. 2002;368(Pt 1):253-61.

21. Li DD, Wang Y, Dai BD, et al. ECM17-dependent methionine/cysteine biosynthesis contributes to biofilm formation in C. albicans [J]. Fungal Genet Biol. 2013:51:50-9.

22. Garcia-Sanchez S, Aubert S, Iraqui I, et al. C. albicans biofilms: a developmental state associated with specific and stable gene expression patterns [J]. Eukaryot Cell. 2004;3(2):536-45.

23. Li J, Zhang B, Ma T, et al. Role of the inositol polyphosphate multikinase Ipk2 in regulation of Hyphal development, calcium signaling and secretion in C. albicans [J]. Mycopathologia. 2017;182(7-8):609-23.

24. Fiorini A, Rosado FR, Bettega EM, et al. C. albicans PROTEIN PROFILE CHAN GES IN RESPONSE TO THE BUTANOLIC EXTRACT OF Sapindus saponariaL [J]. Rev Inst Med Trop Sao Paulo. 2016;58:25.
25. Wisniewski JR, Zougman A, Nagaraj N, et al. Universal sample preparation method for proteome analysis [J]. Nat Methods. 2009:6(5):359-62.

26. Elias JE, Gygi SP. Target-decoy search strategy for increased confidence in large-scale protein identifications by mass spectrometry [J]. Nat Methods. 2007:4(3):207-14.

27. Tyanova S, Temu T, Sinitcyn $P$, et al. The Perseus computational platform for comprehensive analysis of (prote) omics data [J]. Nat Methods. 2016;13(9): 731-40.

28. Ma J, Chen T, Wu S, Yang C, Bai M, Shu K, Li K, Zhang G, Jin Z, He F, Hermjakob H, Zhu Y. iProX: an integrated proteome resource. Nucleic Acids Res. 2019:47(D1):D1211-D1217.

\section{Publisher's Note}

Springer Nature remains neutral with regard to jurisdictional claims in published maps and institutional affiliations.
Ready to submit your research? Choose BMC and benefit from:

- fast, convenient online submission

- thorough peer review by experienced researchers in your field

- rapid publication on acceptance

- support for research data, including large and complex data types

- gold Open Access which fosters wider collaboration and increased citations

- maximum visibility for your research: over $100 \mathrm{M}$ website views per year

At $\mathrm{BMC}$, research is always in progress.

Learn more biomedcentral.com/submissions 